\title{
An empirical equation for shear viscosity of shear thickening fluids
}

DOI:

10.1016/j.molliq.2020.115220

\section{Document Version}

Accepted author manuscript

Link to publication record in Manchester Research Explorer

\section{Citation for published version (APA):}

Shende, T., Niasar, V. J., \& Babaei, M. (2021). An empirical equation for shear viscosity of shear thickening fluids. Journal of Molecular Liquids, 325, [115220]. https://doi.org/10.1016/j.molliq.2020.115220

\section{Published in:}

Journal of Molecular Liquids

\section{Citing this paper}

Please note that where the full-text provided on Manchester Research Explorer is the Author Accepted Manuscript or Proof version this may differ from the final Published version. If citing, it is advised that you check and use the publisher's definitive version.

\section{General rights}

Copyright and moral rights for the publications made accessible in the Research Explorer are retained by the authors and/or other copyright owners and it is a condition of accessing publications that users recognise and abide by the legal requirements associated with these rights.

\section{Takedown policy}

If you believe that this document breaches copyright please refer to the University of Manchester's Takedown Procedures [http://man.ac.uk/04Y6Bo] or contact uml.scholarlycommunications@manchester.ac.uk providing relevant details, so we can investigate your claim.

\section{OPEN ACCESS}




\title{
An empirical equation for shear viscosity of shear thickening fluids
}

\author{
Takshak Shende ${ }^{\mathrm{a}, *}$, Vahid J Niasar ${ }^{\mathrm{a}}$, Masoud Babaei ${ }^{\mathrm{a}, *}$ \\ ${ }^{a}$ The University of Manchester, Department of Chemical Engineering and Analytical \\ Science, Manchester, UK
}

\begin{abstract}
Quantitative modelling of the rheology of non-Newtonian fluids requires significant empirical input due to very complex behaviour of the bulk fluid as a result of particle-scale physics of fluids. The existing rheology models are mainly limited to certain fluid dynamics conditions such as shear rate, shear stress, etc. Adopting Doolittle's free volume theory approach, we have proposed an empirical equation to describe the relative free volumedependent viscosity, the shear stress-dependent viscosity, the shear ratedependent viscosity, and the dimensionless Péclet number-dependent relative viscosity of shear thickening fluids. The proposed formulae predict all rheologically different behaving Newtonian, intermediate shear thinning, shear thickening and extreme shear thinning regimes of shear-thickening fluids. The proposed formulae have been validated against the experimental rheological data of various shear thickening fluids over a range of $\mathrm{pH}$, volume fraction, electrolyte concentration, temperature, and magnetic field. The results suggest that the predicted threshold material parameters of shear thickening fluids help to quantitatively evaluate the effect of vary-
\end{abstract}

\footnotetext{
${ }^{*}$ Corresponding author

Email addresses: takshak.shende@manchester.ac.uk (Takshak Shende), masoud.babaei@manchester.ac.uk (Masoud Babaei)
} 
ing physico-chemical conditions on the rheology of shear thickening fluids. We simulated the flow of a shear thickening fluid, modelled using proposed shear rate-dependent equation, in a 2D staggered porous medium. We observed bimodal distribution of pore-scale shear rate, shear viscosity and velocity in a porous medium.

keywords: Colloidal suspension, Non-Newtonian fluid, Shear thickening fluid, Direct numerical simulation, OpenFOAM, Pore-scale simulation 


\section{Introduction}

The suspension shear-thickening behaviour (i.e. increase in the shear viscosity with an increase in the shear stress/rate showed by colloidal/noncolloidal suspension) [1] has wide applications starting from cement [2], woven fabrics [3], chocolates [4], cornstarch [5] to ceramics [6]. The shearthickening fluids are excellent for shock-absorption due to their high elastic modulus and high dissipation energy $[6,7]$. Smart materials are being developed such as magnetorheological shear thickening fluids [8] which act as actuating fluids to maintain variable flow rate or force transfer scenario in many engineering applications such as artificial joints and breaks [7, 9].

Mixing colloidal particles or polymers (i.e. solute) in a solvent (e.g. water) leads to an increase in the viscosity of the solution, and further increase in the concentration of solute/colloids makes fluid behave like a non-Newtonian fluid. At lower stress, shear thickening fluids show Newtonian behaviour [6]. The fluid shows shear-thinning characteristics once critical stress exceeds, i.e. viscosity of the fluid drops with an increase in stress. The fluid can flow under weak stress and still be able to behave like a gel at rest. The viscosity of the fluid suddenly rises once it reaches critical stress at higher stresses [6]. This behaviour of a fluid is defined as shear thickening, which mostly depends on properties of solute/colloids (shape and size of molecules, volume fraction, etc.) and solvents (viscosity, deformation etc.) $[6,7,10,11]$. Figure 1 shows the typical rheological behaviour of a shear thickening fluid under varying shear stress or shear rate. The typical shear thickening behavior can also be observed at low temperatures ( i.e. temperature $>-40^{\circ} \mathrm{C}$ ) [12].

Interparticle and intermolecular contact forces and hydrodynamic forces significantly contribute towards shear thickening of the suspension in the fluid $[10,13-17]$, the external or internal forces in solution cause displace- 
ment of suspended colloid particles/solute. The force transmits from one colloid particle/solute molecule to the neighbouring colloid particle/solute molecule through intervening fluid. The hydrodynamic interaction between colloid particles/solute and solvent disturb local flow field of all colloids particles/solute molecules $[17,18]$. We note that the intervening fluid (solvent) acts as a lubricant between neighbouring colloids particles/solutes $[1,11]$. Hydrophobic or hydrophilic properties of the colloidal particles/polymer molecules along with hydrodynamic interaction between colloid/solute and solvent determines the relative motion of the particles and effectiveness of the solvent to act as a lubricant. The electrostatics $[19,20]$ and van der Waals forces play a significant role in maintaining the viscosity of the solution $[1,7,10]$. Interparticle forces are dominant at lower stresses; on the contrary, hydrodynamic forces play a vital role at high stresses. Alteration of particles surface chemistry, ionic strength, shape and size affect the interparticle or intermolecular forces, thus viscosity of the solution. The shear induced particle migration and shear banding also affect the shear thinning or thickening of the fluid [5].

Although many empirical models have been developed to characterise shear thinning behaviour of non-Newtonian fluids [18], a single equation which could completely characterise rheological behaviour of shear thickening fluids (See Fig. 1) is meagre. Previously, Gopalakrishnan and Zukoski [21] proposed an empirical equation for the shear viscosity based on thermodynamics and hydrodynamic interaction of shear thickening fluids. The constitutive relation proposed by Wyart and Cates [22] for shear thickening suspension is well accepted constitutive model for shear thickening suspension. Galindo-Rosales et al. [23, 24] developed shear rate-dependent branch of equations based on Cross model [25] to capture first shear thinning, shear thickening and second shear thinning region of shear thickening 

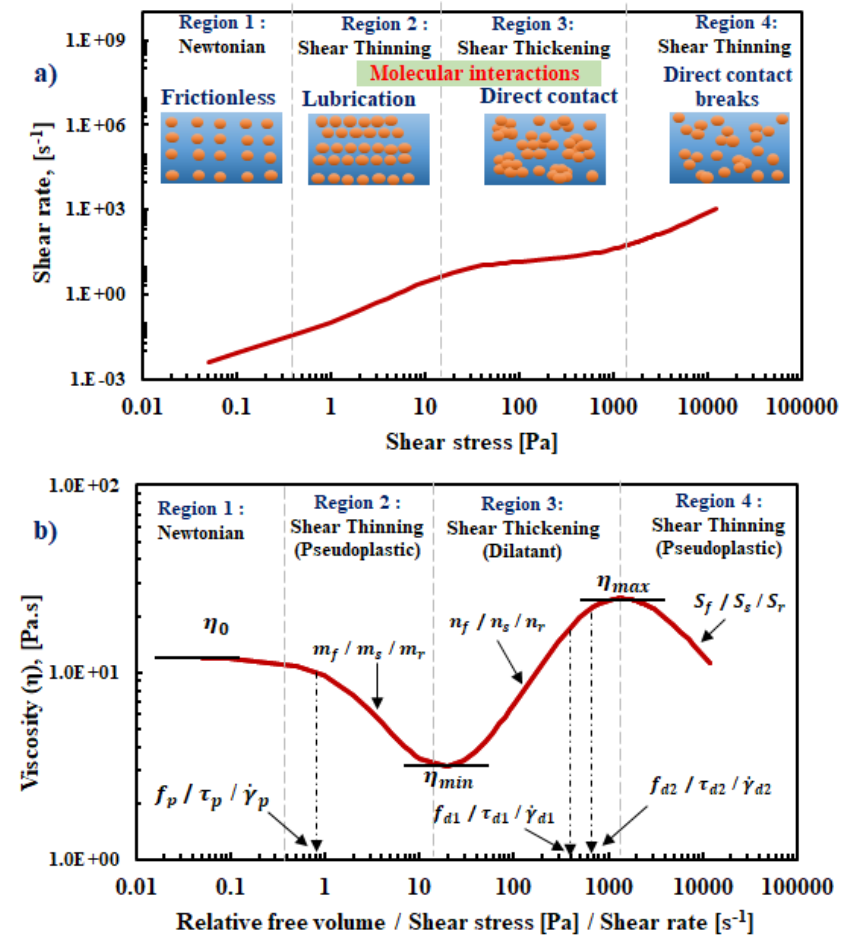

Figure 1: Typical rheological behaviour of a shear thickening fluid under varying relative free volume or shear stress or shear rate. (a) Shear rate - stress relationship, and (b) viscosity as a function of shear stress or shear rate. Typical shear thickening fluid shows Newtonian, shear thinning, shear thickening and second shear thinning regimes. We note that the image is representative and not to scale. 
fluids. Recently, Steller and Iwko [26] proposed an empirical shear stressdependent and shear-rate dependent model for the non-Newtonian fluids based on free volume theory. Steller-Iwko multi-model rheological equation [26] captures the complicated viscosity curve of non-Newtonian fluids. Steller-Iwko [26] modified an empirical equation developed by Doolittle [27] to relate viscosity of the fluid with shear stress and shear rate. Free volume theory proposed by [27] is being extensively used to explain temperature-dependent viscosity variation observed in an amorphous polymer and glass-forming liquids. Williams-Landel-Ferry (WLF) equation [28] developed based on free volume theory of Doolittle [27] has been employed to model time temperature-dependent rheology of polymeric solutions (e.g. polystyrene melts [29], supra-macromolecular polymers [30]).

Free volume theory assumes that the viscosity of the fluid depends on the free space available for molecules to move in a total volume. The relative free-space available for molecules or particles depends on the stresses generated in the fluids [27]. Adopting Doolittle approach, the interlink between relative free space, viscosity and stress can be established to empirically define viscosity as a function of the relative free volume of shear thickening fluids.

The absence of a single rheological equation, which could characterise all regimes of shear thickening fluids (see Fig 1) and could be utilised for porescale fluid flow numerical simulation, motivated us to develop an empirical equation based on free volume theory that models rheological characteristics of shear thickening fluids using measurable parameters and identify threshold parameters of shear thickening fluid. We propose an empirical equation that relates the shear viscosity of shear thickening fluid with a relative free volume. The proposed free volume-based equation is then utilised to develop a shear stress-dependent rheological equation and shear rate- 
dependent rheological equation for shear thickening fluids. The proposed equations are validated against experimental data. Further, we simulate the flow of shear thickening fluid, using the proposed equation, through a 2D micromodel porous medium.

\section{Mathematical Formulations}

The shear viscosity of non-Newtonian fluids can be defined using constitutive equation of generalised Newtonian fluids (GNF) [31, 32]. The shear stress-dependent viscosity functions $(\eta(\tau))$ define viscosity as a function of the magnitude of deviatoric stress tensor (i.e. $\boldsymbol{\tau}=2 \eta(\tau) \boldsymbol{D}$ ) $[26,33-35]$. The shear rate-dependent viscosity function $(\eta(\dot{\gamma}))$ defines viscosity as a function of second invariant of rate-of-deformation tensor (i.e. $\boldsymbol{\tau}=2 \eta(\dot{\gamma}) \boldsymbol{D})[31,32]$. Here, the magnitude of shear stress is $|\tau|=\sqrt{\frac{\boldsymbol{\tau}: \boldsymbol{\tau}}{2}}[33]$, the magnitude of shear-rate is $|\dot{\gamma}|=\sqrt{\frac{\dot{\gamma}: \dot{\gamma}}{2}}[32]$, $\boldsymbol{D}=\frac{1}{2} \dot{\gamma}=\frac{1}{2}\left(\nabla \mathbf{u}+(\nabla \mathbf{u})^{T}\right)$, and $\mathbf{u}$ is velocity vector. Most of the GNF model equations are empirical equations and have their limitations [31, 32]. Amongst commonly used GNF equations (i.e. Power-law, Bingham, Herschel-Bulkley, Carreau, Cross [31, 32]) in the literature, Cross equation [25] is considered to be deduced from physical basis (i.e. the rate kinetics of rupture and formation of linkage between the particles of the polymers) [23-25]

The shear stress-dependent Meter model equation (Eq. 1) $[33,36]$ is a logistic equation which describes S-shape type curve (i.e. Newtonian plateau at high and low shear values and slope at intermediate shear values) of many shear-thinning fluids.

$$
\eta=\eta_{\infty}+\frac{\eta_{0}-\eta_{\infty}}{1+\left(\frac{\tau}{\tau_{p}}\right)^{\alpha-1}}
$$


130

here, $\eta[\mathrm{Pa} \cdot \mathrm{s}]$ is the shear viscosity; $\eta_{0}[\mathrm{~Pa} \cdot \mathrm{s}]$ is the zero shear viscosity of the Newtonian region of fluid; $\eta_{\infty}[\mathrm{Pa} \cdot \mathrm{s}]$ is the infinite shear viscosity. $\alpha$ is a Meter model exponent which represents the slope. $\tau_{p}$ is the threshold shear stress at which viscosity value is $\frac{\eta_{0}+\eta_{\infty}}{2}$.

We modified the equation proposed by Meter as follows that captures the typical viscosity curve of the shear thickening fluid over a range of shear stress values (see Fig 1). We note that the proposed equation follows the similar type of approach as adopted by Galindo-Rosales et al. [23, 24],

$$
\eta=\eta_{\min }+\frac{\eta_{0}-\eta_{\min }}{1+\left(\frac{\tau}{\tau_{p}}\right)^{m_{s}}}+\frac{\left(\eta_{\max }-\eta_{\min }\right)\left(\frac{\tau}{\tau_{d 1}}\right)^{n_{s}}}{\left(1+\left(\frac{\tau}{\tau_{d 2}}\right)^{n_{s}}\right)^{S_{s}}}
$$

here, $\eta[\mathrm{Pa} \cdot \mathrm{s}]$ is the shear viscosity; $\eta_{0}[\mathrm{~Pa} \cdot \mathrm{s}]$ is the zero shear viscosity of the Newtonian region of fluid (region 1 ); $\eta_{\min }[\mathrm{Pa} \cdot \mathrm{s}]$ is the minimum viscosity of the intermediate shear thinning region (region 2) of fluid (i.e. minimum shear viscosity); $\eta_{\max }[\mathrm{Pa} \cdot \mathrm{s}]$ is the maximum viscosity of the shear thickening region of fluid (i.e. maximum shear viscosity). $m_{s}$ is an exponent which represents slope of the intermediate shear thinning region of fluid (region 2). $n_{s}$ is an exponent which represents slope of the shear thickening region of fluid; $S_{s}$ is an exponent which represents slope of the extreme shear thinning region of fluid (region 4$). \tau_{p}[\mathrm{~Pa}]$ is the shear stress at which viscosity of the solution drops to $\frac{\eta_{0}+\eta_{\min }}{2}$ in the intermediate shear thinning region (region 2). $\tau_{d 1}[\mathrm{~Pa}]$ and $\tau_{d 2}[\mathrm{~Pa}]$ are shear stresses at which viscosity of the solution drops to intermediate values between $\eta_{\min }$ and $\eta_{\max }$ in the shear thickening region (region 3 ). The viscosity at $\tau_{d 1}$ is $\eta_{\min }+\frac{\eta_{0}-\eta_{\min }}{1+\left(\frac{\tau_{d 1}}{\tau_{P}}\right)^{m_{s}}}+\frac{\left(\eta_{\max }-\eta_{\min }\right)}{\left(1+\left(\frac{\tau_{d 1}}{\tau_{d 2}}\right)^{n_{s}}\right)^{S_{s}}}$ and the viscosity at $\tau_{d 2}$ is 
${ }_{131} \eta_{\min }+\frac{\eta_{0}-\eta_{\min }}{1+\left(\frac{\tau_{d 2}}{\tau_{P}}\right)^{m_{s}}}+\frac{\left(\eta_{\max }-\eta_{\min }\right)\left(\frac{\tau_{d 2}}{\tau_{d 1}}\right)^{n_{s}}}{2^{S_{s}}}$. Fig. 1b shows rheological

material parameters of the shear stress-dependent viscosity.

\section{Free-Volume Theory}

Most of the non-Newtonian fluid solutions are mixture of solute or colloidal particles (e.g. cornstarch, xanthan-gum) in a solvent (e.g. water). Note that colloids (e.g. silica nano-particles) are phase separated and insoluble molecules in the solution, whereas solute (e.g. polymers) dissolve in the solvent. The small molecular size of the solvent in the solution helps solvent to act as a lubricant between solute/colloid molecules of larger size. The shear thinning or shear thickening behaviour observed in the non-Newtonian fluids depend highly on the thickness of the solvent's lubricant layer between solute/colloid particles molecules [6, 11, 37]. Interaction between solute or colloid molecules is relatively frictionless (i.e. in equilibrium ) at lower stress [6], and this may be the reason for Newtonian behaviour at a lower shear rate or shear stress (as in Region 1, Fig 1). An increase in the stress decreases frictionless interaction between solute/colloids molecules [1, 6, 15]; and solvent effectively acts as a lubricant between solute/colloids molecules. Solvent, being a lubricant, resists direct interaction between solute/colloids molecules. The viscous nature of the solvent streamlines solute/colloids molecules and increases the efficient transport of a solute/colloids in the solvent $[6,10]$. The flow of a solvent is mostly laminar between solute/colloids molecules. This causes a drop in viscosity of a solution with an increase in shear stress (see Region 2 in

Fig 1). Effectiveness of a solvent to act as a lubricant ceases at sufficiently higher shear stresses. Coagulation of solute/colloids molecules forms hydro cluster $[6,10,11,37,38]$ in the solution due to direct contact between so- 
lute/colloids molecules. The viscosity of the fluid increases abruptly at high stresses due to the larger size of the coagulated hydro cluster (see Region 3 in Fig 1). However, at very high shear stresses direct contact between coagulated solute/colloids molecules breaks [39]. This leads to smaller size hydro clusters and separated solute/colloids molecules which decrease the viscosity of the solution (see Region 4 in Fig 1). The viscosity of the fluid could also decrease due to rupture or erosion of the hydro-clusters or particles. The presence of artefact in the suspension may lead to the slipping of the particles if the colloidal suspension is solid-like. This may cause shear thinning at high shear values. We note that hydrodynamic and interparticle (intermolecular) interactions between solute/colloids and solvent strongly affect the relative free volume of solute/colloids and solvent and, in turn, viscosity of the solution is changed.

Macleod (1923) [40] was first to postulate that the viscosity of a fluid is influenced by the free space available for molecules to move in a total volume. He suggested that crowding of more molecules in small space will increase the viscosity of the fluid as it will increase the cohesive force between molecules and decrease the required time for molecules to move out of contact. The free space theory proposed by Macleod was further developed by Doolittle [27] in which he defined free-space in a liquid as space arises due to liquid's total thermal expansion without phase change.

Doolittle defined relative free space as in Eq. 3,

$$
\frac{V_{f}}{V_{0}}=\frac{V-V_{0}}{V_{0}}
$$

${ }_{179}$ Where $\frac{V_{f}}{V_{0}}$ is a relative free space for a single substance; $V_{f}$ is a volume of free space per gram at any temperature; $V_{0}$ is a volume of one gram of liquid extrapolated to absolute zero temperature without change of phase; $V$ is a volume of one gram of liquid at any temperature. Doolittle [27] 
the free volume space as in Eq. 4,

$$
\eta=A e^{\left(\frac{B}{V_{f} / V_{0}}\right)}
$$

Here, $A$ and $B$ are material constants for a single substance. Although Doolittle validated Eq. 4 for the temperature-dependent decrease in viscosity of a Newtonian fluid, he noted that pressure and temperature could change relative free space in the liquid. Although Doolittle equation (i.e. Eq. 4) can not define rheology of shear thickening fluids, we could extend the free volume theory of Doolittle to model rheology of non-Newtonian fluids, specifically shear thickening fluids.

\subsection{Relative free-volume dependent viscosity}

Extending Doolittle free volume theory [27], we propose an empirical equation (Eq. 5) that relates the relative free volume $(f)$ with the shear viscosity $(\eta)$ of shear thickening fluids. We assume that (a) relative free volume of the solution changes due to hydrodynamic and inter molecular (or particle) forces; (b) fluid flow and deformation, thermal variation, external and internal pressure, molecular movement due to chemical reactions generate these hydrodynamic and inter molecular (or particle) forces in a solution; (c) the change in relative free volume is proportional to the local stresses (shear, normal, or thermal); (d) $V_{f}$ is free volume at any stress, $V_{0}$ is volume of liquid at a zero stress and absolute zero temperature without phase change, $V$ is volume of liquid at any stress, and the relative free 
204 volume is $f=\frac{V-V_{0}}{V_{0}}=\frac{V_{f}}{V_{0}}$. Eq. 5 has the following form similar to Eq. 2 :

$$
\eta=\eta_{\min }+\frac{\eta_{0}-\eta_{\min }}{1+\left(\frac{f}{f_{p}}\right)^{m_{f}}}+\frac{\left(\eta_{\max }-\eta_{\min }\right)\left(\frac{f}{f_{d 1}}\right)^{n_{f}}}{\left(1+\left(\frac{f}{f_{d 2}}\right)^{n_{f}}\right)^{S_{f}}}
$$
${ }_{213}$ (region 3$)$. The viscosity at $f_{d 1}$ is $\eta_{\min }+\frac{\eta_{0}-\eta_{\min }}{1+\left(\frac{f_{d 1}}{f_{p}}\right)^{m_{f}}}+\frac{\left(\eta_{\max }-\eta_{\min }\right)}{\left(1+\left(\frac{f_{d 1}}{f_{d 2}}\right)^{n_{f}}\right)^{S_{f}}}$
${ }_{214}$ and the viscosity at $f_{d 2}$ is $\eta_{\min }+\frac{\eta_{0}-\eta_{\min }}{1+\left(\frac{f_{d 2}}{f_{p}}\right)^{m_{f}}}+\frac{\left(\eta_{\max }-\eta_{\min }\right)\left(\frac{f_{d 2}}{f_{d 1}}\right)^{n_{f}}}{2^{S_{f}}}$.
Fig. 1b shows rheological material parameters of a relative free volume-

here, $f_{p}$ is a relative free volume of the fluid at which viscosity of the solution drops to $\frac{\eta_{0}+\eta_{\min }}{2}$ in the intermediate shear thinning region (region $2) ; m_{f}$ is an exponent which represents slope of the intermediate shear thinning region of fluid (region 2). $n_{f}$ is an exponent which represents slope of the shear thickening region of fluid; $S_{f}$ is an exponent which represents slope of the extreme shear thinning region of fluid (region 4). $f_{d 1}$ and $f_{d 2}$ are relative free volumes of fluid at which viscosity of the solution drops to an intermediate value between $\eta_{\min }$ and $\eta_{\max }$ in the shear thickening region dependent viscosity.

\subsection{Shear stress-dependent viscosity}

The relative free volume in the liquid depends on the mobility of the molecules [26] (i.e. colloids or solutes and solvents). The size and shape of molecules, interaction amongst fluid molecules, spatial arrangement and intermolecular forces between molecules, and nature of bulk fluid flow determines rheology of the fluid $[6,10,11,15]$. An interaction amongst molecules (i.e. colloids, solute, solvent etc.) during fluid flow, thermal changes, and external/internal pressure generate stresses in the liquid. The relative free 
space in the liquid changes under the influence of these stresses. We propose a non-linear empirical relationship between stresses in the liquid with a relative free volume as in Eq. 6,

$$
f=\left(\frac{\tau}{\tau_{0}}\right)^{m}
$$

Here, $\tau_{0}[\mathrm{~Pa}]$ and $m$ are material constants of the fluid. By substituting Eq. 6 in Eq. 5, we obtain the shear stress-dependent rheological model (Eq. 2) for a shear thickening fluid. The shear rate of the fluid will be as in Eq. 7

$$
\dot{\gamma}=\frac{\tau}{\left(\eta_{\min }+\frac{\eta_{0}-\eta_{\min }}{1+\left(\frac{\tau}{\tau_{p}}\right)^{m_{s}}}+\frac{\left(\eta_{\max }-\eta_{\min }\right)\left(\frac{\tau}{\tau_{d 1}}\right)^{n_{s}}}{\left(1+\left(\frac{\tau}{\tau_{d 2}}\right)^{n_{s}}\right)^{S_{s}}}\right)}
$$

here, $\tau_{p}=\tau_{0}\left(f_{p}\right)^{\frac{1}{m}}, \tau_{d 1}=\tau_{0}\left(f_{d 1}\right)^{\frac{1}{m}}, \tau_{d 2}=\tau_{0}\left(f_{d 2}\right)^{\frac{1}{m}}, m_{s}=m\left(m_{f}\right)$, and $n_{s}=m\left(n_{f}\right)$.

\subsection{Shear rate-dependent viscosity}

We note that the deformation of fluid under stresses alter the relative free volume of the fluid. We propose a non-linear [18] empirical relationship between shear rate with a relative free volume as in Eq. 8

$$
f=\left(\frac{\dot{\gamma}}{\dot{\gamma}_{0}}\right)^{n}
$$

Here, $\dot{\gamma}_{0}\left[\mathrm{~s}^{-1}\right]$ and $n$ are material constants of the fluid. By substituting Eq. 8 in Eq. 5, we obtain a shear rate-dependent rheological model (Eq. 9) 
for shear thickening fluids,

$$
\eta=\eta_{\min }+\frac{\eta_{0}-\eta_{\min }}{1+\left(\frac{\dot{\gamma}}{\dot{\gamma}_{p}}\right)^{m_{r}}}+\frac{\left(\eta_{\max }-\eta_{\min }\right)\left(\frac{\dot{\gamma}}{\dot{\gamma}_{d 1}}\right)^{n_{r}}}{\left(1+\left(\frac{\dot{\gamma}}{\dot{\gamma}_{d 2}}\right)^{n_{r}}\right)^{S_{r}}}
$$

241

242

here, $\dot{\gamma}_{p}=\dot{\gamma}_{0}\left(f_{p}\right)^{\frac{1}{n}}, \dot{\gamma}_{d 1}=\dot{\gamma}_{0}\left(f_{d 1}\right)^{\frac{1}{n}}, \dot{\gamma}_{d 2}=\dot{\gamma}_{0}\left(f_{d 2}\right)^{\frac{1}{n}}, m_{r}=n\left(m_{f}\right)$, and $n_{r}=n\left(n_{f}\right) ; \dot{\gamma}_{p}\left[\mathrm{~s}^{-1}\right]$ is a shear rate at which viscosity of the solution drops to $\frac{\eta_{0}+\eta_{\min }}{2}$ in the intermediate shear thinning region (region 2). $m_{r}$ is an exponent which represents slope of the intermediate shear thinning region of fluid (region 2). $n_{r}$ is an exponent which represents slope of the shear thickening region of fluid; $S_{r}$ is an exponent which represents slope of the extreme shear thinning region of fluid (region 4). $\dot{\gamma}_{d 1}\left[\mathrm{~s}^{-1}\right]$ and $\dot{\gamma}_{d 2}\left[\mathrm{~s}^{-1}\right]$ are shear rate at which viscosity of the solution drops to an intermediate values between $\eta_{\min }$ and $\eta_{\max }$ in the shear thickening region (region 3). The viscosity at $\dot{\gamma}_{d 1}$ is $\eta_{\min }+\frac{\eta_{0}-\eta_{\min }}{1+\left(\frac{\dot{\gamma}_{d 1}}{\dot{\gamma}_{p}}\right)^{m_{r}}}+\frac{\left(\eta_{\max }-\eta_{\min }\right)}{\left(1+\left(\frac{\dot{\gamma}_{d 1}}{\dot{\gamma}_{d 2}}\right)^{n_{r}}\right)^{S_{r}}}$ and the 251 viscosity at $\dot{\gamma}_{d 2}$ is $\eta_{\min }+\frac{\eta_{0}-\eta_{\min }}{1+\left(\frac{\dot{\gamma}_{d 2}}{\dot{\gamma}_{p}}\right)^{m_{r}}}+\frac{\left(\eta_{\max }-\eta_{\min }\right)\left(\frac{\dot{\gamma}_{d 2}}{\dot{\gamma}_{d 1}}\right)^{n_{r}}}{2^{S_{r}}}$. Fig. 1b shows rheological material parameters of a shear rate-dependent viscosity.

\subsection{Péclet number-dependent relative viscosity}

Stokes-Einstein-Sutherland equation (Eq. 10) defines diffusivity $(D)$ of the molecules/particles with a thermal energy $k_{B} T$ in a solvent of viscosity $\eta_{s}[41,42]$ as

$$
D=\frac{k_{B} T}{6 \pi \eta_{s} a}
$$

where, $k_{B}[\mathrm{~J} / \mathrm{K}]$ is the Boltzmann's constant, $T[\mathrm{~K}]$ is the absolute temperature, $a[\mathrm{~m}]$ is the radius of the molecules/particles. Péclet number 
$(P e)$, also known as dimensionless shear rate [41], relates the magnitude of thermal motion of molecules with a motion caused by shear. Péclet number as defined in Eq. 11 quantifies strength of the shear force compared to the Brownian forces in the fluid [41],

$$
P e=\frac{\dot{\gamma}}{\frac{D}{a^{2}}}=\frac{6 \pi \eta_{s} \dot{\gamma} a^{3}}{k_{B} T}
$$

We obtain the dimensionless Péclet number-dependent relative viscosity of the shear thickening fluid (Eq. 12) by diving Eq. 9 over $\eta_{s}$ and substituting Eq. 11 for the shear rate in Eq. 9,

$$
\frac{\eta}{\eta_{s}}=\frac{\eta_{\min }}{\eta_{s}}+\frac{\frac{\eta_{0}}{\eta_{s}}-\frac{\eta_{\min }}{\eta_{s}}}{1+\left(\frac{P e}{P e_{p}}\right)^{m_{r}}}+\frac{\left(\frac{\eta_{\max }}{\eta_{s}}-\frac{\eta_{\min }}{\eta_{s}}\right)\left(\frac{P e}{P e_{d 1}}\right)^{n_{r}}}{\left(1+\left(\frac{P e}{P e_{d 2}}\right)^{n_{r}}\right)^{S_{r}}}
$$

here, $P e_{p}$ is a Péclet number at which relative viscosity of the solution drops to $\frac{1}{2}\left(\frac{\eta_{0}}{\eta_{s}}+\frac{\eta_{\min }}{\eta_{s}}\right)$ in the intermediate shear thinning region (region 2). $P e_{d 1}$ and $P e_{d 2}$ are Péclet number at which relative viscosity of the solution drops to an intermediate values between $\frac{\eta_{\min }}{\eta_{s}}$ and $\frac{\eta_{\max }}{\eta_{s}}$ in the shear thickening region (region 3). $P e<<1$ shows domination of Brownian motion in the fluid while $P e>>1$ suggests that the microstructure is distorted by flow forces [41, 43]. Thus, arrangement of the molecules is highly dependent on Péclet number and by that viscosity of the fluid [43].

\subsection{Conversion to an existing model}

Eq. 2 converts to the shear stress-dependent Meter model (Eq. 1) [33, 36] by substituting $\eta_{\infty}=\eta_{\min }=\eta_{\max }$ and $m_{s}=\alpha-1$. Similarly, Eq. 9 converts to the shear rate-dependent Cross model equation [25] (Eq. 13), 
${ }_{278}$ by substituting $\eta_{\infty}=\eta_{\min }=\eta_{\max }$ and $\left(\frac{1}{\dot{\gamma}_{p}}\right)^{m_{r}}=C$.

$$
\eta=\eta_{\infty}+\frac{\eta_{0}-\eta_{\infty}}{1+C \dot{\gamma}^{m_{r}}}
$$

279

280

290

$t$ is the time, and $\boldsymbol{\tau}$ is the stress tensor as defined in Eq. 16

$$
\boldsymbol{\tau}=\eta(\dot{\gamma})\left(\nabla \mathbf{u}+(\nabla \mathbf{u})^{T}\right)
$$

${ }_{292}$ Where $\eta(\dot{\gamma})$ is the shear viscosity of a shear thickening fluid, which is 293 a function of shear rate. The magnitude of the rate of strain tensor for 294 shear-dominated flow is as follows

$$
\dot{\gamma}=\sqrt{\frac{\dot{\gamma}: \dot{\gamma}}{2}}=\sqrt{2 \mathbf{D}: \mathbf{D}}
$$


here, $\dot{\gamma}=\left(\nabla \mathbf{u}+(\nabla \mathbf{u})^{T}\right)$ and $\mathbf{D}=\frac{1}{2} \dot{\gamma}$

The shear viscosity $[\eta(\dot{\gamma})]$ of a shear rate-dependent model is as defined in Eq. 9.

\subsubsection{Numerical domain, boundary condition and solver}

We utilised finite volume method based OpenFOAM $\mathrm{C}++$ libraries [44, 45] for solving Eq. 14 and Eq. 15 in conjunction with a shear ratedependent model equation (Eq. 9). We simulated flow of a shear thickening fluid through a $2 \mathrm{D}$ circular staggered micromodel porous medium over a range of constant injection velocity $\left(10^{-7} \mathrm{~m} / \mathrm{s}-0.1 \mathrm{~m} / \mathrm{s}\right)$. The constant injection velocity was applied at the right boundary. The numerical domain had a uniform velocity of zero at the beginning of the simulation. The no-slip condition was deployed at the boundary wall of the micromodel. The snappyHexMesh module and blockMesh module of OpenFOAM were employed to generate a fine hexagonal mesh in a porous media domain.

The PIMPLE algorithm with nOuterCorrectors of 5 was employed to maintain stability and accuracy of shear thickening fluid flow simulations. The Gauss linear uncorrected scheme of OpenFOAM was employed to discretise Laplacian term of governing equations. Gauss linear scheme was used to discretise the gradient term and divergence term. The Gauss linear scheme uses standard finite volume Gaussian integration. The time scheme of the momentum equation was discretised using the second-order implicit backward method. SmoothSolver with a Gauss-Seidel smoother was used to determine velocity profile and Generalised Geometric-Algebraic Multi Grid (GAMG) solver with diagonal incomplete-Cholesky (symmetric) smoother was employed to estimate pressure field. The Courant number was maintained below 0.1 using time-step of $10^{-6} \mathrm{~s}$. The convergence criteria were set to $10^{-6}$ for both the velocity and pressure fields. We used Paraview 
5.7.0 software to visualise and post process the shear thickening fluid flow simulation data.

\section{Results and Discussion}

\subsection{Validation of the shear-stress dependent equation}

We utilised rheological data of Cwalina et al. [46] and Laun [47] to validate the proposed shear stress-dependent model (Eq. 2). Cwalina et al. [46] investigated shear stress-dependent rheological characteristics of silica nanoparticles (diameter $520 \mathrm{~nm}$ ) plus cubic aluminosilicate zeolites dispersed in a polyethylene glycol suspending fluid at colloidal volume fraction $(\phi)$ of $0.445,0.518$ and 0.507 . Laun [47] scrutinized shear stress-dependent viscosity of a styrene-ethyl-acrylate-copolymer-latex of diameter $250 \mathrm{~nm}$ dispersed in the water over range of $\mathrm{pH}$ and $\mathrm{NaCl}$ concentration. We used Excel Solver-GRG nonlinear algorithm [48] to determine material parameters of the proposed rheological equations. The estimated material parameters of the shear thickening fluids of [46] and [47] are as given in Table 1.

Fig 2 and Fig 3 show good fit of the viscosity-shear stress data using Eq. 2 and shear rate-shear stress data using Eq. 7 with experimental measurements of Cwalina et al. [46] ( $\phi$ : 0.445 - 0.518) and Laun [47] (pH: 5.1 - 7; $\mathrm{NaCl}$ Conc.: 0 - $1.14 \mathrm{~g} / \mathrm{L}$ ). The root means square error (RMSE) of the Eq. 2 and Eq. 7 with respect to the experimental data ranged from $5.4 \times 10^{-4}$ to $1.6 \times 10^{-2}$ in Fig 2 and Fig 3, respectively, which suggest statistically good fits of the shear stress-dependent equation with the experiment.

Eq. 2 could capture Newtonian, shear thinning and shear thickening region of the silica nanoparticles based shear thickening fluid of [46] at $\phi$ of 0.445. At $\phi=0.507$, Eq. 2 could capture shear thinning, shear thickening and extreme shear thinning at high shear values (see Fig $2 \mathrm{a}$ and $2 \mathrm{~b}$ ) of 

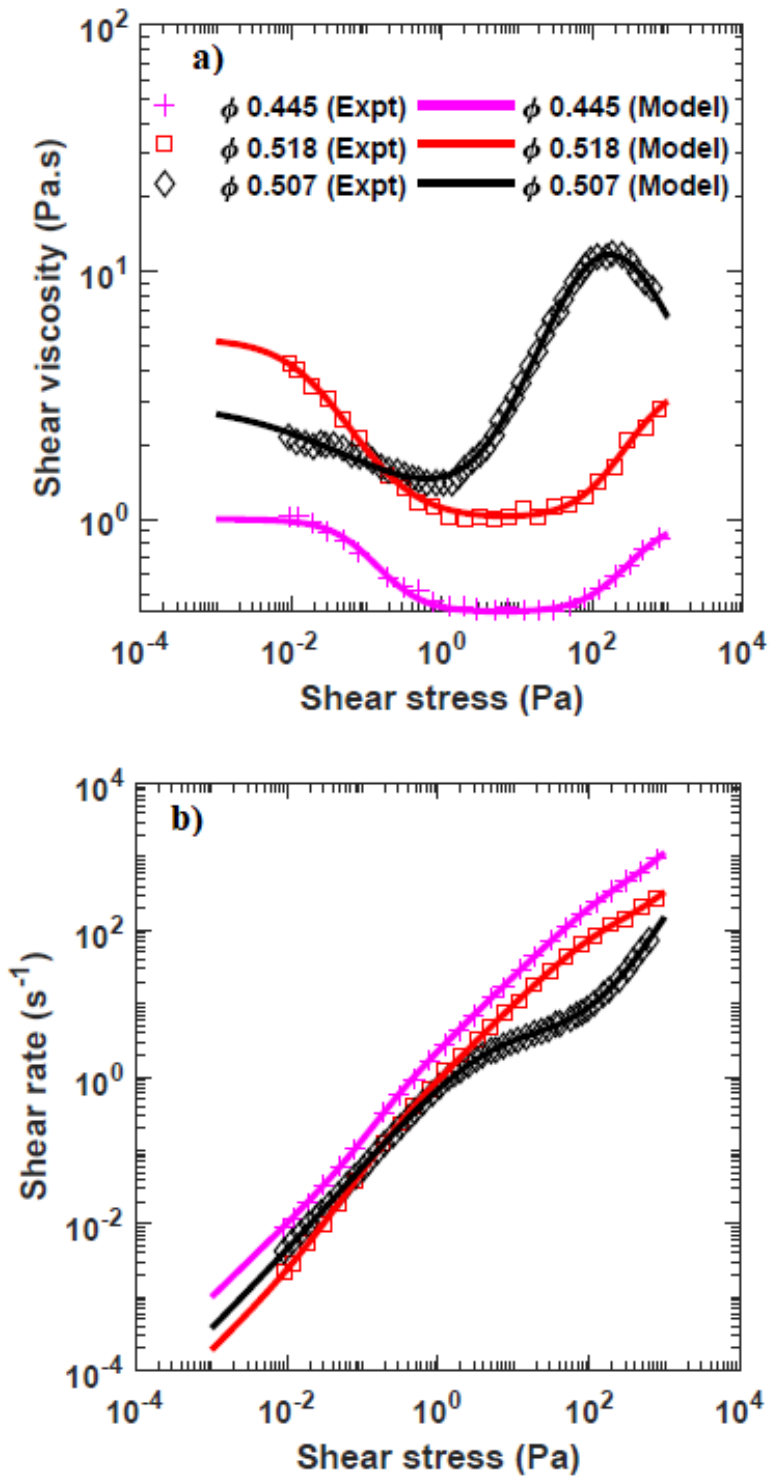

Figure 2: An experimental rheological data of a silica nanoparticles and cubic aluminosilicate zeolites -based shear thickening fluid of [46] over a range of volume fraction $(\phi)$ modelled using a proposed shear stress-dependent model. (a) shear viscosity as a function of shear stress modelled using shear stress-dependent equation (Eq. 2), and (b) shear rate as a function of shear stress modelled using shear stress dependent equation (Eq. 7). The material parameters are given in Table 1. 
Table 1: Estimated material parameters for shear thickening silica nanoparticles-cubic aluminosilicate zeolites based fluid of [46] over range of volume fraction and shear thickening styrene-ethylacrylatecopolymer latex fluid of [47] over range of $\mathrm{pH}$ and $\mathrm{NaCl}$ con-

\begin{tabular}{c|ccc|cccccccc} 
centration. & \multicolumn{10}{c}{ pH of solution $[47]$} & \multicolumn{3}{c}{ NaCl conc $(\mathrm{g} / \mathrm{L})[47]$} \\
\hline & 0.445 & 0.507 & 0.518 & 5.1 & 5.7 & 6.2 & 7 & 0 & 0.287 & 1.14 \\
\hline$\eta_{0}[\mathrm{~Pa} . \mathrm{s}]$ & 1 & 3 & 5.4 & 0.787 & 11.84 & 83.408 & 200 & 120.2 & 50 & 0.2 \\
$\eta_{\min }[\mathrm{Pa} . \mathrm{s}]$ & 0.42 & 1 & 1 & 0.034 & 0.034 & 0.034 & 0.034 & 0.034 & 0.034 & 0.034 \\
$\eta_{\max }[\mathrm{Pa} . \mathrm{s}]$ & 2 & 12 & 4 & 0.72 & 0.260 & 0.170 & 0.09 & 0.15 & 0.23 & 0.52 \\
$\tau_{p}[\mathrm{~Pa}]$ & 0.1 & 0.024 & 0.026 & 0.05 & 0.033 & 0.029 & 0.04 & 0.02 & 0.02 & 0.304 \\
$\tau_{d 1}[\mathrm{~Pa}]$ & 815 & 44 & 490 & 604.6 & 2567 & 6700 & 12847 & 8398 & 3476 & 1120 \\
$\tau_{d 2}[\mathrm{~Pa}]$ & 393 & 146 & 445 & 939 & 3629 & 10196 & 21474 & 14000 & 3041 & 4621 \\
$m_{s}$ & 1.37 & 0.5 & 1 & 1.344 & 2.02 & 2.3 & 2.1 & 2 & 1.9 & 2.845 \\
$n_{s}$ & 1.4 & 1.04 & 1.29 & 1.389 & 1.462 & 1.354 & 1.3 & 1.804 & 1.172 & 0.969 \\
$S_{s}$ & 1 & 1.85 & 1 & 1.261 & 1.194 & 1.255 & 1.004 & 1.347 & 0.9 & 2.005 \\
\hline
\end{tabular}

the shear thickening fluid of [46]. Fig 3 shows that Eq. 2 correctly depicts the effects of the $\mathrm{pH}$ and $\mathrm{NaCl}$ concentration on the rheological behaviour of the styrene-ethyl-acrylate-copolymer-latex fluid. Charge on the particle and degree of dissociation change due to $\mathrm{pH}$ variation in the solution. Addition of $\mathrm{NaCl}$-salt in the solution increases total ion concentration in the fluid. These parameters strongly affect intermolecular interactions (due to van der Waals and electrostatic forces) between colloids and solvents. The relative free volume in the solution changes due to the combined effect of these intermolecular interactions and hydrodynamic forces. Table 1 shows decrease in $\eta_{\max }$ and an increase in $\eta_{0}, \tau_{d 1}$ and $\tau_{d 2}$ with an increase in the $\mathrm{pH}$. On the contrary, Table 1 reports increase in $\eta_{\max }$ and decrease in $\tau_{d 1}$ and $\tau_{d 2}$ with an increase in the electrolyte $(\mathrm{NaCl})$ concentration. We note that the proposed model predicted Newtonian viscosity $\left(\eta_{0}\right)$ of [47] shear thickening fluid solution, which would not have been possible to identify by observation of the rheological data or graph. The predicted 
minimum shear-thinning viscosity $\left(\eta_{\min }\right)$ of $0.034 \mathrm{~Pa}$.s over a range of $\mathrm{pH}$, and $\mathrm{NaCl}$ concentration of [47] indicate that varying hydrogen concentration and ionic strength did not significantly affect the $\eta_{\min }$ of a latex fluid. This suggests that the proposed rheological equation helps to quantitatively correlate rheological behaviour of the shear thickening fluid under varying physiochemical conditions.

\subsection{Validation of the shear-rate dependent equation}

We utilised rheological data of Kalman et al. [49], Maus et al. [50] and Zhang et al. [51] to validate proposed shear rate-dependent model (Eq. 9). Kalman et al. [49] investigated shear rate-dependent rheological characteristics of monodisperse polymethylmethacrylate (PMMA) particles dispersed in polyethylene glycol fluid at colloidal volume fraction $(\phi)$ of $0.40,0.45,0.48$ and 0.49 . Maus et al. [50] measured shear viscosity of the $\alpha, \omega$-Mg carboxylato-polyisoprene (MCPI) in decahydronaphthalene over a range of temperature $\left(25{ }^{\circ} \mathrm{C}, 30{ }^{\circ} \mathrm{C}, 35{ }^{\circ} \mathrm{C}\right)$. Zhang et al. [51] scrutinized shear rate-dependent viscosity of carbonyl iron particles of $5 \mu \mathrm{m}$ mean size and fumed silica particles dispersed in ethylene glycol over a range of magnetic field $(\mathrm{kA} / \mathrm{m})$. The estimated material parameters of the shear thickening fluids of [49], [50], and [51] are as given in Table 2.

Fig 4, Fig 5 and Fig 6 show good fit of the viscosity-shear rate data using Eq. 9 with experimental measurements of Kalman et al. [49] ( $\phi$ : 0.4 - 0.49), Maus et al. (Temp: 25-35 ${ }^{\circ} \mathrm{C}$ ) [50], and Zhang et al. [51] (Magnetic field: $0-300 \mathrm{kA} / \mathrm{m}$ ). The root means square error (RMSE) of the Eq. 9 with respect to the experimental data is ranged from $2 \times 10^{-1}$ to $3 \times 10^{1}$ in Fig 4, Fig 5, and Fig 6 respectively, which suggests a statistically good fit of the shear rate-dependent equation with experiments.

Fig 4 illustrates that the Eq. 9 can encompass all four regions (Newtonian, intermediate shear thinning, shear thickening, and extreme shear 

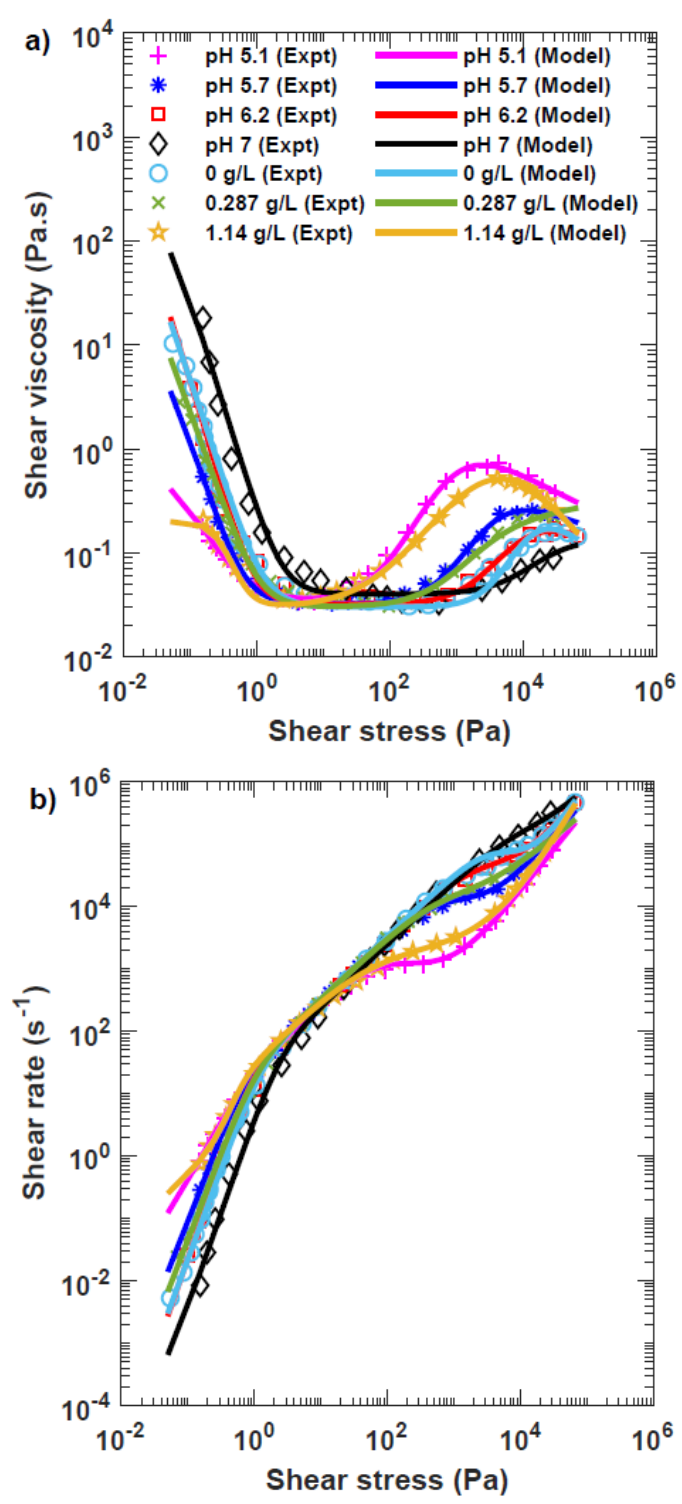

Figure 3: The experimental rheological data of styrene-ethyl-acrylate-copolymer-latex based shear thickening fluid of [47] over a range of $\mathrm{pH}$ and $\mathrm{NaCl}$ concentrations modelled using proposed shear stress-dependent model. (a) shear viscosity as a function of shear stress modelled using shear stress-dependent equation (Eq. 2), and (b) shear rate as a function of shear stress modelled using shear stress dependent equation (Eq. 7). The material parameters are given Table 1 . 


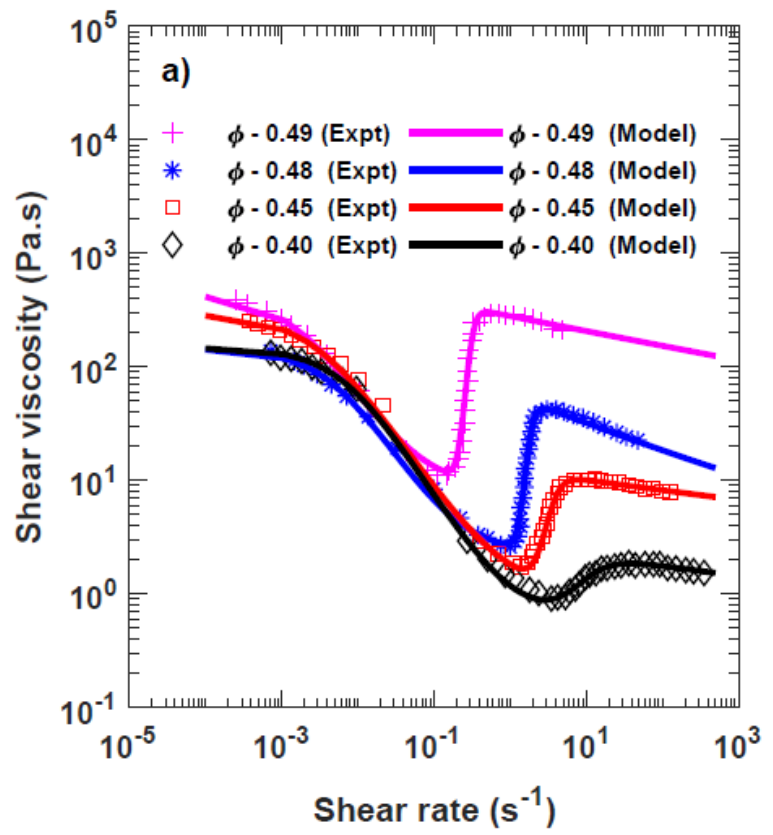

Figure 4: Shear viscosity of monodisprsed polymethylmethacrylate (PMMA) particles based shear thickening fluid of [49] over a range of volume fraction $(\phi)$ modelled using the proposed shear rate-dependent equation (Eq. 9). The material parameters are given Table 2. 


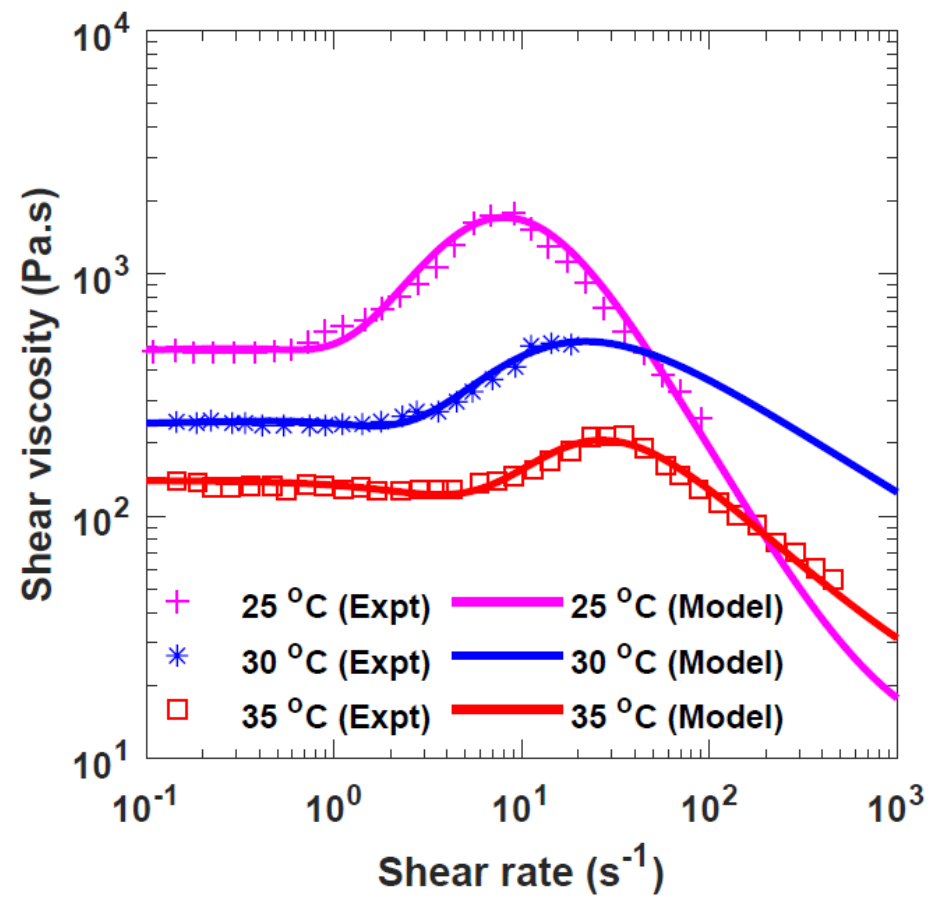

Figure 5: Shear viscosity of $\alpha, \omega$-Mg carboxylato-polyisoprene (MCPI)-based shear thickening fluid of [50] over a range of temperatures modelled using the proposed shear ratedependent equation (Eq. 9). The material parameters are given Table 2. 
Table 2: Estimated material parameters for the shear thickening PMMA particles-based fluid of [49] over a range of volume fraction, the MCPI fluid of [50] over a range of temperature, and the carbonyl iron and silica particles-based fluids of [51] over a range of magnetic field

\begin{tabular}{|c|c|c|c|c|c|c|c|c|c|c|c|}
\hline \multirow[t]{2}{*}{ Parameter } & \multicolumn{4}{|c|}{ Volume fraction $(\phi)[49]$} & \multicolumn{3}{|c|}{ Temperature $\left({ }^{\circ} \mathrm{C}\right)[50]$} & \multicolumn{4}{|c|}{ Magnetic field $(\mathrm{kA} / \mathrm{m})[51]$} \\
\hline & 0.49 & 0.48 & 0.45 & 0.40 & 25 & 30 & 35 & 0 & 100 & 200 & 300 \\
\hline$\eta_{0}[\mathrm{~Pa} . \mathrm{s}]$ & 437 & 143 & 292 & 146 & 477 & 239 & 140 & 20 & 300 & 500 & 800 \\
\hline$\eta_{\min }[\mathrm{Pa} . \mathrm{s}]$ & 8.34 & 2.3 & 1 & 0.60 & 10 & 10 & 10 & 1.5 & 2.0 & 10 & 11 \\
\hline$\eta_{\max }[\mathrm{Pa} . \mathrm{s}]$ & 293 & 42 & 10 & 1.82 & 1770 & 520 & 213 & 25 & 29 & 32 & 45 \\
\hline$\dot{\gamma}_{p}\left[\mathrm{~s}^{-1}\right]$ & 0.00146 & 0.0044 & 0.0028 & 0.0065 & 0.9 & 1.8 & 5 & 2.2 & 0.7 & 1.8 & 1.5 \\
\hline$\dot{\gamma}_{d 1}\left[\mathrm{~s}^{-1}\right]$ & 0.3 & 1.8 & 4 & 10 & 3 & 5.5 & 11 & 961 & 522 & 415 & 420 \\
\hline$\dot{\gamma}_{d 2}\left[\mathrm{~s}^{-1}\right]$ & 0.3 & 1.82 & 3.61 & 10 & 7.8 & 11 & 18.52 & 1095 & 557 & 467 & 442 \\
\hline$m_{r}$ & 1.1 & 1.1 & 1 & 1.1 & 2.1 & 1.9 & 1.5 & 0.9 & 0.82 & 1.3 & 1.3 \\
\hline$n_{r}$ & 10.6 & 8.88 & 4.27 & 2.04 & 1.46 & 1.24 & 1.59 & 6.46 & 7.09 & 5.31 & 7.69 \\
\hline$S_{r}$ & 1.01 & 1.03 & 1.03 & 1.07 & 1.95 & 1.42 & 1.49 & 1.43 & 1.13 & 1.21 & 1.16 \\
\hline
\end{tabular}

thinning) of the PMMA shear thickening fluid over a range of $\phi$ values.

On the contrary, as depicted in Fig 5, intermediate shear-thinning region is absent in the rheological data of [50]. Yet, Eq. 9 is able to correctly capture Newtonian followed by shear thickening and extreme shear-thinning regions of the fluid over a range of temperature values. Further, Table 2 shows constant minimum viscosity $\left(\eta_{\min }\right)$ of the fluid at high shear rate values in the extreme shear thinning region. This suggests that $\eta_{\min }$ also represents the lowest limiting viscosity value of the extreme shear thinning region.

An increase in the $\phi$ of the particles in the solution increased $\eta_{\min }$ and $\eta_{\max }$, and decreased $\dot{\gamma}_{d 1}$ and $\dot{\gamma}_{d 2}$ (see Table 2) of [49]. We note that $\dot{\gamma}_{d 1}$ and $\dot{\gamma}_{d 2}$ have approximately same values for given $\phi$ values of [49]. The slope $\left(m_{r}\right)$ of the intermediate shear thinning region of [49] has same value of 1.1 over a range of $\phi$, except for $\phi=0.45$. These results suggest that the shear rate-dependent rheological model helps predict threshold shear 


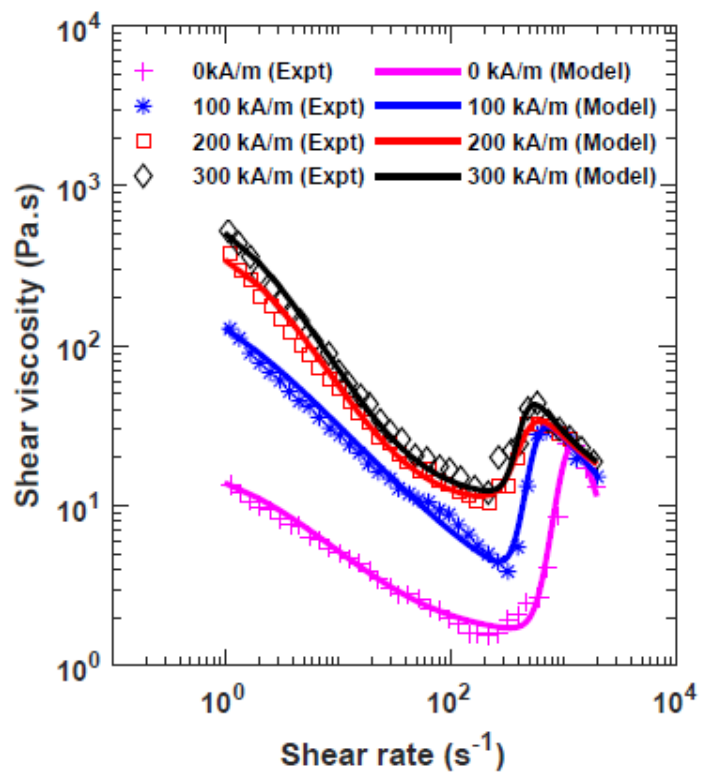

Figure 6: Shear viscosity of carbonyl iron particles and fumed silica particles-based magnetorheological shear thickening fluid of [51] over a range magnetic field modelled using the proposed shear rate-dependent equation (Eq. 9). The material parameters are given in Table 2 .

rate values $\left(\dot{\gamma}_{p}, \dot{\gamma}_{d 1}\right.$, and $\left.\dot{\gamma}_{d 2}\right)$. As predicted, the rheological parameters of [50] show decrease in $\eta_{0}, \eta_{\max }, m_{r}$ and increase in $\dot{\gamma}_{d 1}, \dot{\gamma}_{d 2}, n_{r}$ with increase in the temperature of the shear thickening fluid. We note that increase in the temperature generates thermal stresses, which change the relative free volume in the solution. This leads to decrease in the viscosity of the solution with increase in the temperature. Increase in exposure of the magnetic field $(0-300 \mathrm{kA} / \mathrm{m})$ to the shear thickening fluid of [51] increased $\eta_{0}, \eta_{\min }, \eta_{\max }$ and decreased $\dot{\gamma}_{d 1}$ and $\dot{\gamma}_{d 2}$ (see Table 2 and Fig 6). The shear-rate dependent model (Eq. 9) could estimate $\eta_{\min }$ of the fluid.

These results suggest that the shear stress-dependent equation (Eq. 2) 
Table 3: Estimated material parameters for a shear thickening superball silica fluid of [52] over a range of volume fraction

\begin{tabular}{ccc}
\hline Parameters & \multicolumn{2}{c}{ Volume fraction $(\phi)[52]$} \\
& 0.24 & 0.28 \\
\hline$\frac{\eta_{0}}{\eta_{s}}$ & 1.98 & 2.6 \\
$\frac{\eta_{\text {min }}}{\eta_{s}}$ & 1.96 & 2.53 \\
$\frac{\eta_{\text {max }}}{\eta_{s}}$ & 2.02 & 2.72 \\
$P e_{p}$ & 800 & 700 \\
$P e_{d 1}$ & $4 \times 10^{4}$ & $1.8 \times 10^{4}$ \\
$P e_{d 2}$ & $4.1 \times 10^{4}$ & $2.1 \times 10^{4}$ \\
$m_{r}$ & 2 & 1.5 \\
$n_{r}$ & 1.5 & 1.1 \\
$S_{r}$ & 1.01 & 1.03 \\
\hline
\end{tabular}

and shear rate dependent equation (Eq. 9) capture highly non-linear rheological characteristics of the shear thickening fluid and predict threshold measurable parameters of shear thickening fluids such as threshold shear stress/rate.

\subsection{Validation of the Péclet number-dependent equation}

Fig 7 shows a perfect fit of the experimentally determined Péclet numberdependent relative viscosity of the superball silica fluid of [52] with Eq. 12. The material parameters are given in Table 3. Pe in Fig 7 corresponds to the shear rate of $10^{0} \mathrm{~s}^{-1}$ to $10^{3} \mathrm{~s}^{-1}$. We note that $P e$ takes into account the size of the particle (see Eq. 11). The observed shear thickening behaviour of the fluid at $P e>10^{3}$ suggests a strong dependence of shear thickening on changes in the microstructural arrangement. The $P e_{p}, P e_{d 1}$, $P e_{d 2}$ values obtained using Eq. 12 could be considered as a threshold $P e$ which helps to modulate shear thinning and shear thickening behaviours in non-Newtonian fluid. 

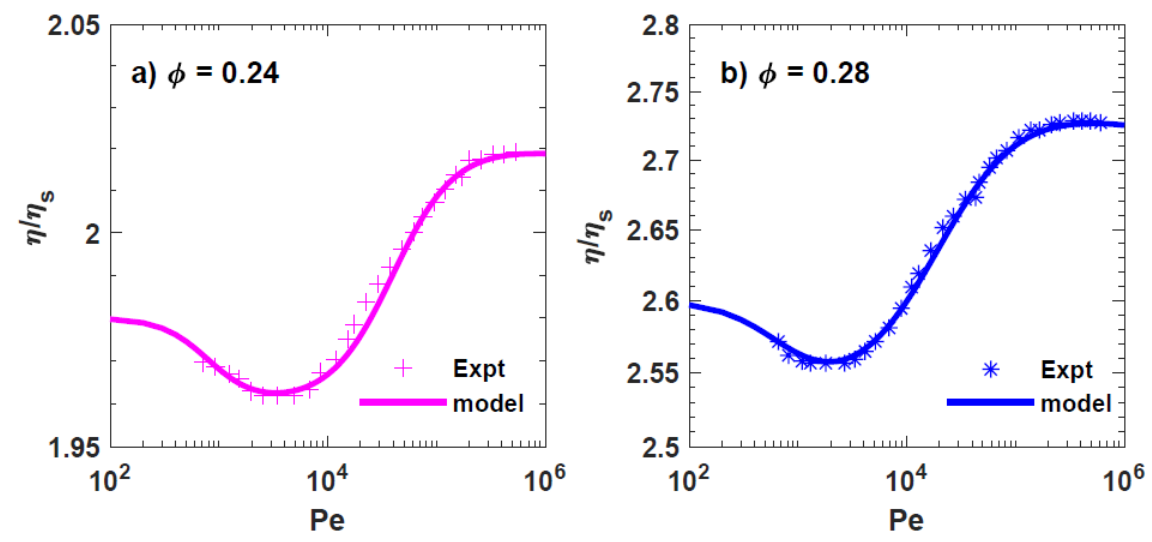

Figure 7: Comparison of the experimentally measured Péclet number-dependent relative viscosity of the superball silica at volume fraction of (a) $\phi=0.24$, and (b) $\phi=0.28$ of [52] with a proposed Péclet Number-dependent equation (Eq. 12).

\subsection{Pore-scale flow simulation}

We simulated the flow of a PMMA particles-based fluid of [49] over a range of constant injection velocity $\left(10^{-7} \mathrm{~m} / \mathrm{s}-0.1 \mathrm{~m} / \mathrm{s}\right)$ in a circular staggered porous medium (domain size: $4.2 \mathrm{~mm} \times 2.4 \mathrm{~mm}$, circle diameter: $262 \mu \mathrm{m}$, pore-throat size: $162 \mu \mathrm{m}$, channel depth: $120 \mu \mathrm{m}$, porosity: 0.7, see Fig 8). The material parameters of PMMA fluid used for simulation are as given in the Table 2 .

Fig 8 shows spatial distribution along with the frequency of the shear rate, shear viscosity and velocity in the $2 \mathrm{D}$ porous medium. Fig $8 \mathrm{~d}, \mathrm{e}, \mathrm{f}$ indicate that the field of shear rate, viscosity and velocity is non-uniformly distributed in the porous media domain. The bi-modal distribution in Fig $8 \mathrm{a}, \mathrm{d}$ suggest that the shear rate profile at the micromodel boundary is different from the central part of the micromodel. The first tall peak in the fig $8 \mathrm{~d}$ is shear rate distribution in the central part, and a small peak at high shear values is shear rate distribution at the boundary. Similar 
to shear rate profile, bi-modal distribution observed in the shear viscosity profile in Fig 8e indicate variation in the distribution of viscosity in the central region and boundary region of the porous medium. Fig 8c imply that the PMMA fluid has higher velocity along the flow direction; further, there exists a no-flow region with a velocity lower than $10^{-5} \mathrm{~m} / \mathrm{s}$ before and after circular blocks of the porous medium.

The average shear rate, the average shear viscosity, and the average velocity of the fluid flow in the porous medium for each simulation over a range of injection velocity are estimated using 'integrate variable' filter of the Paraview 5.7.0 software. Fig 9a compare the average shear rate of the porous medium with an average viscosity of the porous medium over a range of volume fraction and injection rate. Fig 9a depict shear thickening behaviour of the fluid in the porous medium similar to the rheological data. Note that the average values of shear rate and shear viscosity of the porous medium do not overlap with a rheological data of the PMMA fluid measured using a rheometer (i.e. as in Fig 4).

Fig $9 \mathrm{~b}$ shows the non-linear relationship between average velocity and pressure gradient during the flow of PMMA based shear thickening fluid in the 2D staggered porous medium. Further, we can deduce from Fig 9b that with an increase in the volume fraction of the particle in the fluid increases the pressure requirements. The results of direct numerical simulation indicate that the proposed shear rate dependent rheological model for shear thickening fluid can be utilised to gain pore-scale insight of shear thickening fluid flow. In future, we will use the proposed rheological model to numerically simulate two-phase shear thickening fluid flow using volume of fluid method, specifically to understand the shear thickening fluid flow behaviour while displacing Newtonian fluid at pore-scale. 
a)

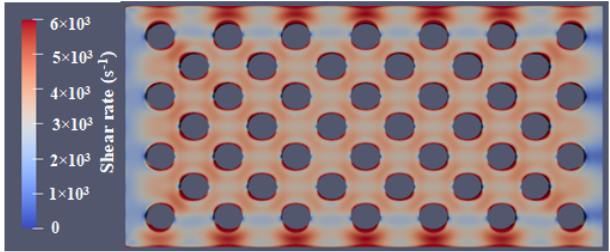

b)

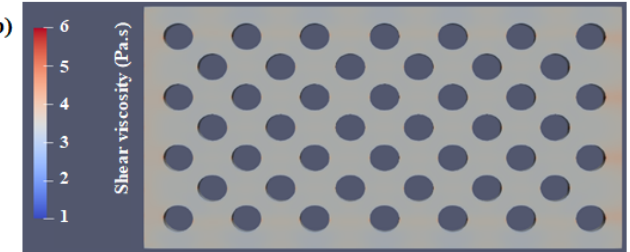

c)

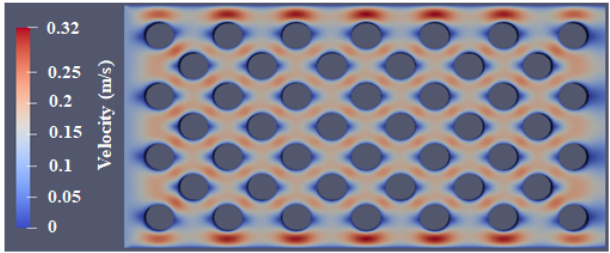

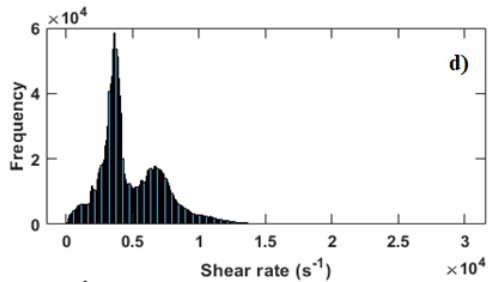
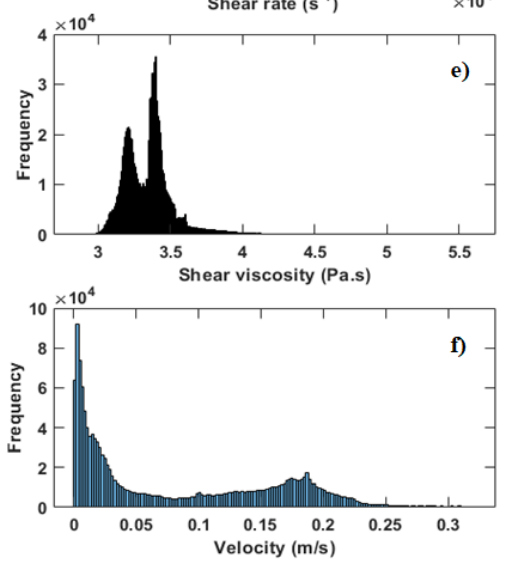

Figure 8: Spatial distribution of a) shear rate (b) shear viscosity (c) velocity and frequency of d) shear rate e) shear viscosity $\mathrm{f}$ ) velocity in the porous media domain during PMMA fluid $(\phi=0.45)$ flow at a constant injection velocity of $0.1 \mathrm{~m} / \mathrm{s}$.
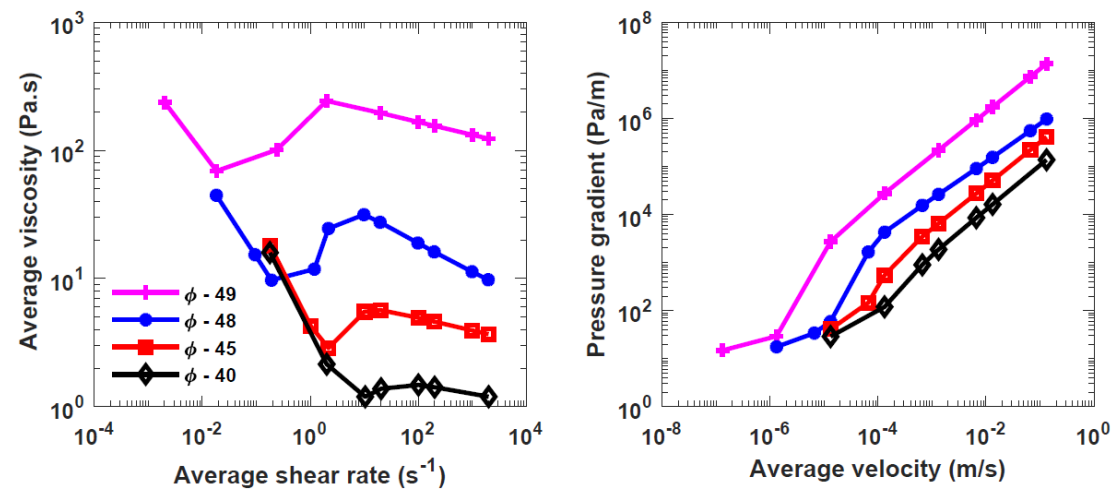

Figure 9: (a) Average shear viscosity - average shear rate, and (b) average velocity pressure gradient over a range of volume fraction $(\phi)$ obtained after simulated flow of PMAA shear thickening fluid of [49] through the 2D staggered porous medium over a range of constant injection rates. 


\section{Conclusions}

The free volume theory proposed by Doolittle is protracted for a shear thickening fluid. An empirical formula is proposed to relate the shear viscosity of shear thickening fluid with a relative free volume of the fluid. The stresses generated in the fluid due to intermolecular forces, hydrodynamic forces, and thermal variation cause a change in the relative free volume. The non-linear relationship between stresses and the relative free volume is used to develop a shear stress-dependent rheological equation for shear thickening fluids which captures different behaviours such as Newtonian, intermediate shear thinning, shear thickening, and the extreme shear-thinning regime of a shear thickening fluid. An equation for the shear rate-dependent viscosity is also formulated using a non-linear relationship between the shear rate and the relative free volume. The proposed formulations convert to the existing Meter model and Cross model. We note that the proposed rheological model equation are empirical generalised Newtonian fluid (GNF) models similar to power-law, Carreau, Cross, Meter models.

The perfect fit of the experimental data of various shear thickening fluid (silica nanoparticles, latex, PMMA particles, MCPI, carbonyl iron particles based shear thickening fluid) with the shear stress-dependent equation and the shear rate-dependent equation over a range of physicochemical properties (volume fraction, $\mathrm{pH}, \mathrm{NaCl}$ concentration, temperature, magnetic field) suggests that the proposed rheological formulations could be utilised to evaluate rheological characteristics of the shear thickening fluid using measurable parameters. The nine material parameters of the proposed formulation help to quantitatively analyse the effect of varying physicochemical conditions on rheology of shear thickening fluids. The proposed equation predicts critical viscosity parameters of shear thickening fluids such as $\eta_{0}, \eta_{\min }, \eta_{\max }$. The threshold shear stress parameters $\left(\tau_{0}, \tau_{p}\right.$, 
$\left.\tau_{d 1}, \tau_{d 2}\right)$, the threshold shear rate parameters $\left(\dot{\gamma}_{0}, \dot{\gamma}_{p}, \dot{\gamma}_{d 1}, \dot{\gamma}_{d 2}\right)$ and the threshold Péclet number parameters $\left(P e_{p}, P e_{d 1}, P e_{d 2}\right)$ of the proposed equations are parameters that modulate the effective transmutation of rheological characteristics (i.e. from Newtonian to shear-thinning followed by shear-thickening regime).

Numerical simulations of PMMA-based fluid (modelled using shear ratedependent equation) flow through $2 \mathrm{D}$ circular staggered porous medium shows that the proposed shear rate dependent equation can be employed for conducting shear thickening fluid flow studies. We observed shear thinning, shear thickening followed by again shear thinning of the PMMA-based fluid flow in $2 \mathrm{D}$ porous medium. The shear rate, shear viscosity and velocity in 2D porous medium has bimodal distribution, which indicate that the flow behavior of the shear thickening fluid at boundary of porous medium is different than in the central part of the porous medium. We will extend this work a) to upscale pore-scale shear viscosity of the shear thickening fluid to continuum scale and b) to simulate two phase shear thickening fluid flow in a porous medium.

\section{Acknowledgement}

T.S. gratefully appreciates Rajashri Shahu Maharaj Foreign Scholarship, Government of Maharashtra, India that has enabled him to undertake $\mathrm{PhD}$ research at the University of Manchester. The authors would like to acknowledge the assistance given by Research IT and the use of the Computational Shared Facility at The University of Manchester. The authors would like to thanks two anonymous reviewers for their constructive comments and suggestions. 


\section{Conflict of interests}

The authors declare that they have no conflict of interest.

\section{References}

[1] A. Singh, R. Mari, M. M. Denn, J. F. Morris, A constitutive model for simple shear of dense frictional suspensions, Journal of Rheology 62 (2) (2018) 457-468.

[2] C. D. Cwalina, N. J. Wagner, Rheology of non-brownian particles suspended in concentrated colloidal dispersions at low particle reynolds number, Journal of Rheology 60 (1) (2016) 47-59.

[3] D. Li, R. Wang, X. Liu, S. Fang, Y. Sun, Shear-thickening fluid using oxygen-plasma-modified multi-walled carbon nanotubes to improve the quasi-static stab resistance of kevlar fabrics, Polymers 10 (12) (2018) 1356.

[4] V. A. Fernandes, A. J. Müller, A. J. Sandoval, Thermal, structural and rheological characteristics of dark chocolate with different compositions, Journal of Food Engineering 116 (1) (2013) 97-108.

[5] A. Fall, F. Bertrand, G. Ovarlez, D. Bonn, Shear thickening of cornstarch suspensions, Journal of Rheology 56 (3) (2012) 575-591.

[6] N. J. Wagner, J. F. Brady, Shear thickening in colloidal dispersions, Physics Today 62 (10) (2009) 27-32.

[7] S. Gürgen, M. C. Kuşhan, W. Li, Shear thickening fluids in protective applications: a review, Progress in Polymer Science 75 (2017) 48-72. 
[8] C. Yang, Z. Liu, M. Yu, X. Bian, The influence of thixotropy on the magnetorheological property of oil-based ferrofluid, Journal of Molecular Liquids (2020) 114425.

[9] S. H. Sadek, H. H. Najafabadi, F. J. Galindo-Rosales, Capillary breakup extensional magnetorheometry, Journal of Rheology 64 (1) (2020) 55-65.

[10] S. Mueller, E. Llewellin, H. Mader, The rheology of suspensions of solid particles, Proceedings of the Royal Society A: Mathematical, Physical and Engineering Sciences 466 (2116) (2010) 1201-1228.

[11] E. Brown, H. M. Jaeger, Shear thickening in concentrated suspensions: phenomenology, mechanisms and relations to jamming, Reports on Progress in Physics 77 (4) (2014) 046602.

[12] Y. Chen, G. Zeng, W. Liu, The effect of surface grafting polymer chains on the shear thickening of hard microsphere suspensions, Colloids and Surfaces A: Physicochemical and Engineering Aspects 555 (2018) 736745 .

[13] N. Y. Lin, B. M. Guy, M. Hermes, C. Ness, J. Sun, W. C. Poon, I. Cohen, Hydrodynamic and contact contributions to continuous shear thickening in colloidal suspensions, Physical Review Letters 115 (22) (2015) 228304.

[14] H. C. Chu, R. N. Zia, The non-newtonian rheology of hydrodynamically interacting colloids via active, nonlinear microrheology, Journal of Rheology 61 (3) (2017) 551-574.

[15] R. Mari, R. Seto, J. F. Morris, M. M. Denn, Shear thickening, frictionless and frictional rheologies in non-brownian suspensions, Journal of Rheology 58 (6) (2014) 1693-1724. 
[16] Y. Thomas Hu, Mechanisms of shear thickening in transient guar network, Journal of Rheology 58 (6) (2014) 1789-1807.

[17] I. E. Zarraga, D. A. Hill, D. T. Leighton Jr, The characterization of the total stress of concentrated suspensions of noncolloidal spheres in newtonian fluids, Journal of Rheology 44 (2) (2000) 185-220.

[18] F. Yilmaz, M. Y. Gundogdu, A critical review on blood flow in large arteries; relevance to blood rheology, viscosity models, and physiologic conditions, Korea-Australia Rheology Journal 20 (4) (2008) 197-211.

[19] W. Kang, X. Hou, C. Chen, S. Shao, X. Zhang, T. Zhu, T. Wang, H. Yang, Study on rheological behavior and salt-thickening mechanism of a synthesized twin-tailed hydrophobically modified polyacrylamide, Journal of Molecular Liquids 294 (2019) 111619.

[20] X. Zhang, H. Yang, P. Wang, T. Zhu, T. Wang, C. Chen, F. Wang, W. Kang, Construction and thickening mechanism of amphiphilic polymer supramolecular system based on polyacid, Journal of Molecular Liquids 286 (2019) 110921.

[21] V. Gopalakrishnan, C. Zukoski, Effect of attractions on shear thickening in dense suspensions, Journal of Rheology 48 (6) (2004) 1321-1344.

[22] M. Wyart, M. Cates, Discontinuous shear thickening without inertia in dense non-brownian suspensions, Physical Review Letters 112 (9) (2014) 098302.

[23] F. J. Galindo-Rosales, F. J. Rubio-Hernández, A. Sevilla, An apparent viscosity function for shear thickening fluids, Journal of NonNewtonian Fluid Mechanics 166 (5-6) (2011) 321-325. 
[24] F. J. Galindo-Rosales, F. J. Rubio-Hernández, A. Sevilla, R. H. Ewoldt, How dr. malcom m. cross may have tackled the development of "an apparent viscosity function for shear thickening fluids", Journal of Non-Newtonian Fluid Mechanics 166 (23-24) (2011) 1421-1424.

[25] M. M. Cross, Rheology of non-newtonian fluids: a new flow equation for pseudoplastic systems, Journal of Colloid Science 20 (5) (1965) 417-437.

[26] R. Steller, J. Iwko, New generalized newtonian fluid models for quantitative description of complex viscous behavior in shear flows, Polymer Engineering \& Science 58 (8) (2018) 1446-1455.

[27] A. K. Doolittle, Studies in newtonian flow. ii. the dependence of the viscosity of liquids on free-space, Journal of Applied Physics 22 (12) (1951) 1471-1475.

[28] M. L. Williams, R. F. Landel, J. D. Ferry, The temperature dependence of relaxation mechanisms in amorphous polymers and other glassforming liquids, Journal of the American Chemical Society 77 (14) (1955) 3701-3707.

[29] D. Parisi, J. Ahn, T. Chang, D. Vlassopoulos, M. Rubinstein, Stress relaxation in symmetric ring-linear polymer blends at low ring fractions, Macromolecules.

[30] S. C. Boothroyd, D. M. Hoyle, T. C. McLeish, E. Munch, R. Schach, A. J. Smith, R. L. Thompson, Association and relaxation of supramacromolecular polymers, Soft Matter 15 (26) (2019) 5296-5307.

[31] R. B. Bird, R. Armstrong, O. Hassager, Fluid mechanics, dynamics of polymeric liquids, Vol. 1 (1987). 
[32] R. B. Bird, W. E. Stewart, E. N. Lightfoot, Transport Phenomena, John Wiley \& Sons, 2007.

[33] D. M. Meter, R. B. Bird, Tube flow of non-Newtonian polymer solutions: Part I. Laminar flow and rheological models, AIChE Journal 10 (6) (1964) 878-881.

[34] G. W. Peters, J. F. Schoonen, F. P. Baaijens, H. E. Meijer, On the performance of enhanced constitutive models for polymer melts in a cross-slot flow, Journal of Non-Newtonian Fluid Mechanics 82 (2-3) (1999) 387-427.

[35] S. Matsuhisa, R. B. Bird, Analytical and numerical solutions for laminar flow of the non-Newtonian Ellis fluid, AIChE Journal 11 (4) (1965) $588-595$.

[36] T. Shende, V. J. Niasar, M. Babaei, Effective viscosity and reynolds number of non-newtonian fluids using meter model, Rheologica Acta (2020) 1-11.

[37] A. Boromand, S. Jamali, B. Grove, J. M. Maia, A generalized frictional and hydrodynamic model of the dynamics and structure of dense colloidal suspensions, Journal of Rheology 62 (4) (2018) 905-918.

[38] O. Cheal, C. Ness, Rheology of dense granular suspensions under extensional flow, Journal of Rheology 62 (2) (2018) 501-512.

[39] J. Fusier, J. Goyon, X. Chateau, F. Toussaint, Rheology signature of flocculated silica suspensions, Journal of Rheology 62 (3) (2018) 753771.

[40] D. Macleod, On a relation between the viscosity of a liquid and its 
${ }_{648}$ coefficient of expansion, Transactions of the Faraday Society 19 (July)

$649 \quad(1923) 6-16$.

650

651

652

653

[41] J. Mewis, N. J. Wagner, Colloidal suspension rheology, Cambridge University Press, 2012.

[42] J. Van der Werff, C. De Kruif, J. Dhont, The shear-thinning behaviour of colloidal dispersions: II. Experiments, Physica A: Statistical Mechanics and its Applications 160 (2) (1989) 205-212.

[43] J. F. Brady, J. F. Morris, Microstructure of strongly sheared suspensions and its impact on rheology and diffusion, Journal of Fluid Mechanics 348 (1997) 103-139.

[44] H. Jasak, A. Jemcov, Z. Tukovic, et al., Openfoam: A c++ library for complex physics simulations, in: International workshop on coupled methods in numerical dynamics, Vol. 1000, IUC Dubrovnik Croatia, 2007, pp. 1-20.

[45] C. J. Greenshields, Openfoam user guide. version 6, OpenFOAM Foundation Ltd July.

[46] C. D. Cwalina, K. J. Harrison, N. J. Wagner, Rheology of cubic particles in a concentrated colloidal dispersion suspending medium, AIChE Journal 63 (3) (2017) 1091-1101.

[47] H. M. Laun, Rheological properties of aqueous polymer dispersions, Die Angewandte Makromolekulare Chemie: Applied Macromolecular Chemistry and Physics 123 (1) (1984) 335-359.

[48] G. Kemmer, S. Keller, Nonlinear least-squares data fitting in excel spreadsheets, Nature protocols 5 (2) (2010) 267. 
[49] D. Kalman, J. Schein, J. Houghton, C. Laufer, E. Wetzel, N. Wagner, Polymer dispersion based shear thickening fluid-fabrics for protective applications, Proceeding of SAMPE (2007) 3-7.

[50] C. Maus, R. Fayt, R. Jérôme, P. Teyssie, Shear thickening of halatotelechelic polymers in apolar solvents, Polymer 36 (10) (1995) 20832088.

[51] X. Zhang, W. Li, X. Gong, Study on magnetorheological shear thickening fluid, Smart Materials and Structures 17 (1) (2008) 015051.

[52] J. R. Royer, G. L. Burton, D. L. Blair, S. D. Hudson, Rheology and dynamics of colloidal superballs, Soft Matter 11 (28) (2015) 5656-5665. 